\title{
An Overview on the Portuguese Quality of Electricity Supply Code Revision 2013: The Continuity of Supply and Power Quality Perspective
}

\author{
S. Faias ${ }^{1,2,3}$, R. Castro ${ }^{3,4}$ and J. Esteves ${ }^{1}$ \\ ${ }^{1}$ Entidade Reguladora dos Serviços Energéticos (ERSE) \\ Rua Dom Cristóvão da Gama, 1 - 3º 1400 - 113 Lisboa (Portugal) \\ Phone number:+351 213033 200, e-mail: SFaias@erse.pt, JEsteves@erse.pt \\ ${ }^{2}$ Lisbon Engineering Superior Institute (ISEL) \\ Rua Conselheiro Emídio Navarro, 1, 1950-062 Lisboa (Portugal) \\ ${ }^{3}$ INESC ID \\ Rua Alves Redol, 9, 1000-029 Lisboa (Portugal) \\ ${ }^{4}$ Instituto Superior Técnico (IST) - University of Lisbon \\ Av. Rovisco Pais, 1, 1049-001 Lisboa (Portugal), e-mail: rcastro@tecnico.ulisboa.pt
}

\begin{abstract}
.
New legislation, published in September and October 2012, transferred to the Portuguese national regulatory authority for energy services (ERSE) the authority to prepare and approve the national regulatory code on the quality of electricity supply. As a consequence, ERSE promoted a revision of the quality of supply code, which came in force in January 2014. This paper presents an overview on the Portuguese quality of electricity supply code revision, highlighting the main topics addressed during this revision, in what concerns continuity of supply and voltage quality aspects. The quality of electricity supply code revision created an opportunity to adjust the quality of supply requirements to the current networks performances, to promote a change on the continuity of supply performance analysis, from a network operators perspective to a customers perspective, and to increase the share of responsibilities between network operators and users.
\end{abstract}

\section{Key words}

Electricity sector regulation, quality of service, continuity of supply, power quality.

\section{Introduction}

The liberalization of the electricity sector establishes the separation of basic functions of electricity generation, transmission, distribution and supply (or retailing). However, while in the generation and supply functions a potential for competition exists, in the transmission and distribution networks, their natural monopoly nature requires regulation to induce optimal price and quality of service [1]-[3].
The quality of service regulation, also referred to as quality of electricity supply regulation, with its three dimensions, continuity of supply (network reliability and availability), power or voltage quality (characteristics of the supply voltage) and commercial quality (timeliness in dealing with customers' requests) provides a balance between customers' willingness to pay network tariffs and their expectations on minimum levels of quality of service. This topic became more relevant with the evolution from a rate-of-return economic regulation, in which total utility costs were fully recouped by the tariff, to a price-cap regulation, wherein, with the objective of improving the utilities' economic efficiency, their allowed revenues are capped in a level that does not necessarily reflect the actual costs, leading firms to avoid investments and consequently to decrease the quality of service provided to customers [2], [4], [5].

This quality of electricity supply regulation can be exercised with recourse to a set of direct and indirect instruments such as the definition of minimum standards for the continuity of supply indicators and respective individual monetary compensations, financial incentive schemes, implementation of national voltage quality monitoring programmes, regular reporting and dissemination of the network performance and national and international benchmarking [2], [3], [6], [7].

In order to simulate a competitive environment and contribute to the quality of electricity supply performance improvement of regulated companies, the different direct and indirect instruments should be periodically adjusted in accordance with the evolution of the networks performance. In this regard, the Portuguese regulatory 
authority for energy services (ERSE) promoted a revision of the quality of electricity supply code during 2013.

The objective of this paper is to present an overview on the Portuguese quality of electricity supply code revision, in what concerns the continuity of supply and power quality aspects. In this sense, the paper describes the direct and indirect instruments used on the regulation of the quality of electricity supply in Portugal and the main changes introduced by the 2013 code revision, as well as the substantiation for those changes. A description of the steps taken and initiatives promoted by ERSE during this code revision is also included in the paper.

\section{Quality of Electricity Supply Regulation in Portugal}

In Portugal, the first steps towards the electricity sector liberalization started in 1995. As a result, with the objective of defining the minimum quality of service standards that utilities should provide to customers and the practices used to monitor the compliance with those standards, the government published the first Portuguese code on the quality of electricity supply in the year 2000 . This code, comprising the continuity of supply, voltage quality and commercial quality aspects, was revised in the years 2003 and 2006 [8].

Until 2012, the role of the Portuguese regulatory authority on the quality of electricity supply topic was limited to the supervision of compliance with the quality of electricity supply code and to the definition of regulatory incentives. The main direct and indirect instruments used in Portugal for the quality of electricity supply regulation are described next.

\section{A. Continuity of Supply \\ 1) Indicators for the Continuity of Supply Characterization}

The regular operation of distribution networks can be disrupted by several internal (malfunctions of network equipment) or external sources (as for instance extreme atmospheric phenomena) that usually result in interruptions of the customers electricity supply.

The measurement of actual continuity of supply levels through indicators and standards constitutes one of the basic instruments for regulating continuity of supply [2]. In the Portuguese quality of electricity supply code, a set of indicators to measure the number and duration of long term interruptions (over 3 minutes) are defined for low voltage (LV), medium voltage (MV), high voltage (HV) and extra high voltage (EHV). Those main indicators are:

Transmission network:

- TIE - Equivalent duration of interruptions, per year;

- ENS - Energy not supplied due to interruptions, per year;
- $\quad$ SAIFI EHV - Average number of interruptions per delivery point, per year;

- $\quad$ SAIDI EHV - Average duration of interruptions per delivery point, per year;

- SARI - Average time to restore the supply after an interruption;

Distribution networks:

- $\quad$ TIEPI MV - Average duration of interruptions in medium voltage per installed capacity, per year;

- END - Energy not distributed due to interruptions, per year;

- $\quad$ SAIDI MV - Average duration of interruptions in medium voltage per customer, per year;

- $\quad$ SAIFI MV - Average number of interruptions in medium voltage per customer, per year;

- SAIDI LV - Average duration of interruptions in low voltage per customer, per year;

- $\quad$ SAIFI LV - Average number of interruptions in low voltage per customer, per year.

The SAIDI and SAIFI indicators are used in most European countries to characterize the continuity of supply. They are mainly focused in the domestic customer perspective, as they are weighted by the number of customers. In addition, in Portugal and Spain, TIEPI MV is used to characterize the continuity of supply of industrial customers, since it is weighted by the installed capacity, and used for distribution network planning purposes by the operators [9].

\section{2) Standards and Individual Monetary Compensations}

Standards for continuity of supply indicators and respective individual monetary compensations resulting from noncompliance with the standards are considered in the quality of electricity supply code. Those standards, expressed in the form of limits to the number and duration of interruptions experienced by each customer per year, represent a commitment of the company with their customers to maintain a certain level of quality of supply. Whenever individual limits are exceeded, customers must be informed and monetary compensations must be automatically paid. The total amount of monetary compensations annually paid to customers is not supported by tariffs. This may act as an incentive to operator to improve the continuity of supply performance along the years.

Considering that the demography, as well as technical characteristics of the distribution network, is not homogeneous along the country, different standards are defined according to three kinds of areas, urban, suburban and rural. In urban areas it is assumed that the distribution network mainly comprises underground cables in LV and MV and, as such, more demanding standards are prescribed. On the other hand, standards for rural areas are less demanding, since the respective MV and LV networks are characterized by long overhead lines exposed to natural elements. Sub-urban areas 
represent a transition zone between rural and urban areas. In this sense, standards for continuity of supply indicators are in between those defined for urban and rural areas.

\section{3) Incentive for the Continuity of Supply in the Distribution Network}

In complement to standards and individual monetary compensations, incentive schemes comprising revenue increases (rewards) and decreases (penalties) can be developed. Portugal is considered one of the pioneers in Europe regarding the implementation of an incentive for the continuity of supply in the distribution network [6], [10].

ERSE implemented this incentive scheme in 2003 in order to stimulate a performance increase in the MV distribution network. The incentive scheme is based on historical values of the energy not distributed $\left(\mathrm{END}_{\mathrm{REF}}\right)$ indicator. As presented in Fig. 1, a dead band $\left(\mathrm{END}_{\mathrm{REF}} \pm \Delta \mathrm{V}\right)$ is used to avoid the incentive activation when small performance improvement or deterioration is experienced. On the other hand, in order to avoid overstating the impact of the incentive on the company economic results, maximum amount of reward (RQSmáx) and penalty (RQSmin) are defined. Reward and penalty limits are symmetric and, at moment, its value is fixed at 5 million euro. When the performance improvement or deterioration is placed between the dead band boundaries and the reward and penalty limits, the amount of the incentive is computed based on the valorization of the energy not distributed (VEND), 1.5 euro/kWh.

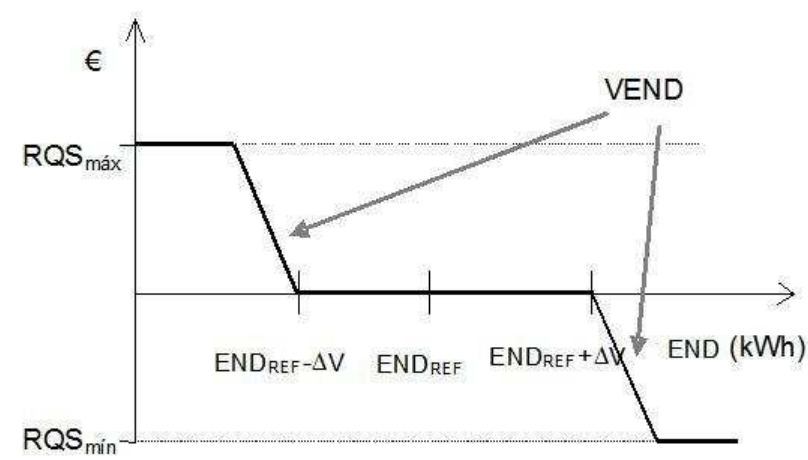

Fig. 1. Incentive scheme for the continuity of supply in MV network.

\section{B. Voltage Quality}

The quality of electricity supply code establishes that network operators must monitor the following voltage characteristics and voltage events in their networks:

- Frequency;

- Supply voltage variations;

- Dips;

- Unbalances;

- Flicker;

- Harmonics.
Under regular conditions of operation, network operators must maintain supply voltage characteristics in compliance with the limits defined in the international standard EN 50160. However, because until the 2010 edition, the scope of this standard was restricted to MV and LV levels, the Portuguese quality of electricity supply code has an annex with the definition of the voltage characteristics limits for the $\mathrm{EHV}$ and $\mathrm{HV}$ networks.

Regarding voltage quality monitoring, the quality of electricity supply code also establishes the obligation of the network operators to develop voltage quality monitoring programs. Those programs should include the monitoring of $100 \%$ of the EHV network delivery points, in every 2 years, $100 \%$ of the HV/MV substations, in every 4 years, and two different MV/LV transformers by municipality, in every 4 years. The duration of each monitoring campaign can vary from one week to one year.

In practice, network operators have adopted more demanding targets for voltage quality monitoring than those required by the quality of electricity supply code [11], [12]. For instance, transmission and distribution network operators, on a voluntary basis, have adopted the strategy of installing permanent voltage quality monitoring devices in all new or refurbished $\mathrm{EHV} / \mathrm{HV}$ and $\mathrm{HV} / \mathrm{MV}$ substations.

Besides the voltage quality monitoring program, network operators must perform a measurement of the voltage characteristics whenever a customer complains.

\section{Reporting and Benchmarking Quality of Electricity Supply}

One of the indirect instruments of the quality of electricity supply regulation is the systematic publication of information about networks performance and benchmarking those results between different companies [3].

In this regard, the present Portuguese quality of electricity supply code requires network operators to publish an annual report including information about their performance, expressed by different indicators defined for continuity of supply, voltage quality and commercial quality, and including information about number and amount of monetary compensations paid to customers that result from noncompliance with minimal quality standards. Also a description of the events that led to the main interruptions occurred over the year must be included in the report. The report on the quality of electricity supply of each company must be available on its website.

In addition, an annual report comprising the evaluation of the utilities' performance is published by the national regulatory authority. This report is made available on its website and disseminated by different stakeholders, namely customer associations. 
In what concerns benchmarking the continuity of supply performance, only international benchmarking is possible since the Portuguese mainland distribution network is operated by one large utility that supplies almost all customers. In this regard, ERSE participates in the elaboration of the European benchmarking reports published by the Council of European Energy Regulators (CEER).

\section{Audits to the Quality of Electricity Supply Information Systems}

The national regulatory authority promote biannual audits to the quality of electricity supply information systems of the network operators in order to assess the integrity and traceability of their procedures in what concerns interruptions registration, computation of indicators and individual monetary compensations, as well as the voltage quality data management. Also the compliance with the quality of electricity supply code, concerning systematic publication of networks performance information is audited.

These audits are performed by independent consultants, according to guidelines defined by the national regulatory authority. ERSE is also responsible to follow the progress of the audits and to provide inputs for the final report of the audit. When deemed appropriate, ERSE can recommend consultants to perform additional queries to the audited system.

The costs of the audits to the quality of electricity supply information systems are supported by the network tariffs.

\section{Main Changes in the 2013 Revision of the Quality of Electricity Supply Code}

As already referred to, in spite of the first Portuguese code on the quality of electricity supply was published back in the year 2000, until 2012 the role of Portuguese regulatory authority on this topic was limited to the supervision of compliance with the quality of electricity supply code and to the definition of regulatory incentives. However, new legislation, published in September and October 2012, transferred to ERSE the authority to prepare and approve the national regulatory framework on the quality of electricity supply. In this regard, during 2013, ERSE promoted a revision of the quality of electricity supply code, identifying the next three main drivers for this code revision:

- Adjust the electricity quality of supply requirements to the current networks performance;

- Increase the share of responsibilities between network operators and users;

- Adapt the regulation to the supply services liberalization.

During the quality of electricity supply code revision, initiated in January 2013, ERSE identified the different stakeholders (customers, network operators, suppliers, academics and other specialists) and promoted a set of meetings with the objective of collecting their proposals. In addition, in April 2013, ERSE organized a workshop dedicated to continuity of supply and power quality subjects with the participation of some international specialists. In June, ERSE published a first proposal for the new quality of electricity supply code, promoted a public consultation and started the collection of comments to improve that first code proposal. The final version of the code was published in November 2013 and came into force in January 2014.

It should be referred that in this code revision process, beyond the proposals received from the different stakeholders, several valuable inputs gathered from the different technical reports produced by CEER were also included, as for instance the different editions of the Benchmarking Report on the Electricity Quality of Supply and some of the Guidelines of Good Practices, as well as other technical reports and international standards published by CENELEC and IEC.

During quality of electricity supply code revision, the different stakeholders identified the need to maintain the participation and interactivity level promoted by ERSE, in what concerns the quality of supply topic. In this sense, a working group to follow the application of the new quality of electricity supply code and to gather inputs for future code revision processes was created.

\section{A. Continuity of Supply}

From the three aspects of the quality of electricity supply, the continuity of supply is the one for which customers are more sensitive. In this sense, one of the objectives identified for this code revision was to promote a change on the continuity of supply performance analysis, from a network operators perspective to a customers perspective [13]. This change of perspective was implemented, for instance, through the obligation of the distribution operators to report all the interruptions that affected their network users, independently from the origin of those interruptions, or by the introduction of new continuity of supply indicators, such as the momentary average interruption frequency index (MAIFI), in order to measure the number of short interruptions (less than 3 minutes) experienced by customers [14].

Another example of this change from the network operators perspective to the customers perspective is the fact that, from now on, in Azores and Madeira islands groups, the continuity of supply performance indicators and system standards must also include the interruptions with origin in generation failures.

The process of the exceptional events classification was also subjected to some changes in this quality of electricity supply code revision. With the new code, all the exceptional events (those whose responsibility is not from the network operators [15]) must be approved by the regulatory authority, based on evidence gathered by the network operators to prove that the origin of the interruption was outward the network, was non- 
predictable and that it was non-economically efficient to avoid its occurrence.

Following the need to adjust the electricity quality of supply requirements to the current networks performance, identified as one of the three main drivers for the quality of electricity supply code revision, the system and individual standards on the continuity of supply were updated (last update was in 2006). The update was more ambitious for the rural area standards (usually with less demanding requirements) in order to define standards closer to those established for the urban area.

In the new quality of electricity supply code a second component of the existing incentive scheme for the increase of the continuity of supply for MV distribution network was also introduced. This new component aims at reducing the gap between average continuity of supply performance of the Portuguese system and the continuity of supply experienced by the customers with worst service supply.

Still regarding continuity of supply, it was also introduced a new limit to the annual monetary compensation paid by network operators to customers when individual standards are exceeded. This new limit corresponds to $100 \%$ of the customer annual network tariff (value of the previous year) and it was defined as result of a benchmarking with other European countries [9].

\section{B. Power Quality}

Voltage quality is an important aspect of the quality of electricity supply that network operators provide to their connected customers. When voltage quality is poor, problems such as reduced life-time, loss of efficiency, flickering lights and even explosion or fire can arise in the use of electrical appliances and equipment.

Larger or industrial customers are those to whom these issues are most noticeable. However, the characteristics of their loads, namely the respective electric current waveform, can also be a source of perturbations that affect the quality of the voltage supplied. In this sense, in order to increase the share of responsibilities between network operators and users, the new quality of electricity supply code evolved from a voltage quality perspective, only focused on the consequences, to a power quality perspective, also committed with the identification and mitigation of the sources.

Since the Portuguese quality of electricity supply code adopted the standard EN 50160 to define the characteristics and limits for the supplied voltage, some of the changes introduced to this standard by CENELEC in the 2010 edition were considered as inputs for the code revision. This was the case of widening the standard scope to the HV network and an adaption of the table used for registration and classification of voltage dips.
Another change introduced in the new code refers to a need for harmonization with the international practice of calculating the equivalent voltage dip when poly-phase events occur. In this case, the method proposed in IEC 61000-4-30 standard was adopted for the Portuguese quality of electricity supply code.

In the previous Portuguese quality of electricity supply code, monitoring voltage swells was not mandatory. As such, due to the potential impact of this issue on the electrical devices life-time, the obligation to record and classify voltage swells was also introduced.

In addition, in what concerns power quality, recognizing the need to increase the knowledge about the network performance, it was introduced the obligation of the network operators to submit a bi-annual plan for the power quality monitoring to be approved by the regulatory authority. Furthermore, the results of the power quality monitoring plans must be published in the network operators web page, disaggregated by monitored network point. Beyond these obligations, some new targets for the number of network points covered by power quality monitoring plans were defined. This is the case of the EHV network, wherein $100 \%$ of the delivery points must be covered by permanent monitoring devices until the end of the year 2016, and also the case of HV and MV networks, in which, at least $20 \%$ of the HV/MV substations must be covered by permanent monitoring or annual monitoring campaigns until the end of 2014.

\section{Conclusions}

In this paper, an overview on the Portuguese quality of electricity supply code revision promoted by ERSE during 2013 was presented.

The quality of electricity supply code revision created an opportunity to adjust the quality of supply requirements to the current networks performance, to promote a change on the continuity of supply performance analysis, from a network operators perspective to a customers perspective, and to increase the share of responsibilities between network operators and users.

This code revision process was also an excel opportunity to involve most stakeholders in an enlarged compromise and to bring their different perspectives to a comprehensive and participated debate. This approach to the revision code process was prized by the stakeholders and the need to maintain this interactivity beyond the 2013 code revision was identified. In this sense, a working group to follow the application of a new quality of electricity supply code and to gather inputs for future code revision processes was created.

Finally, it is worth to mention the valuable inputs gathered from the different technical reports produced by CEER, as well as some technical reports and international standards published by CENELEC and IEC that most enriched the final version of the quality of electricity supply code, in force since January 2014. 


\section{Disclaimer}

The text in this paper represents the personal opinion of the authors and not necessarily the opinion of the Portuguese regulatory authority for energy services (ERSE).

\section{References}

[1] J. Román, J. Gómez, A. Muñoz, and J. Peco, "Regulation of distribution network business," IEEE Trans. Power Delivery, vol. 14, pp. 662-669, Apr. 1999.

[2] V. Ajodhia, and R. Hakvoort, "Economic regulation of quality in electricity distribution networks," Utilities Policy, vol. 13, pp. 211-221, Sep. 2005.

[3] D. Giannakis, T. Jamasb, and M. Pollitt, "Benchmarking and incentive regulation of quality of service: an application to the UK electricity distribution networks," Energy Policy, vol. 33, pp. 2256-2271, Nov. 2005.

[4] V. Ajodhia, L. Schiavo, and R. Malaman, "Quality regulation of electricity distribution in Italy: an evaluation study," Energy Policy, vol. 34, pp. 1478-1486, Sep. 2006.

[5] E. Fumagalli, P. Garrone, and L. Grilli, "Service quality in the electricity industry: the role of privatization and managerial behavior," Energy Policy, vol. 35, pp. 62126224, Dec. 2007.

[6] E. Fumagalli, L. Lo Schiavo, and F. Delestre, Service Quality Regulation in Electricity Distribution and Retail, Berlin: Springer, 2007.

[7] CEER - Council of European Energy Regulators, "Guidelines of Good Practice on the Implementation and Use of Voltage Quality Monitoring Systems for Regulatory Purposes", Brussels, Dec. 2012.
[8] ERSE - Entidade Reguladora dos Serviços Energéticos, Electricity Liberalisation [Online]. Available: http://www.erse.pt/eng/Paginas/ERSE.aspx

[9] CEER - Council of European Energy Regulators, "5th CEER Benchmarking Report on the Quality of Electricity Supply (2011)", Brussels, Apr. 2012.

[10] C. Growitsch, T. Jamasb, C. Müller, and M. Wissner, "Social cost-efficient service quality - Integrating customer valuation in incentive regulation: Evidence from the case of Norway", Energy Policy, vol. 38, pp. 25362544, May. 2010.

[11] L. Campos Pinto and A. Tavares, "Power Quality of Supply Characterization in the Portuguese Electricity Transmission Grid", International Conference on Renewable Energies and Power Quality - ICREPQ'13, Bilbao, Mar. 2013.

[12] A. Lebre, F. Bastião, N. Melo, L. Jorge, P. Veloso and A. Blanco, "Power Quality in the Portuguese Distribution Network", 21st International Conference on Electricity Distribution - CIRED, Frankfurt, Jun. 2011.

[13] S. Faias and J. Esteves, "Continuity of Supply in the Portuguese Distribution Network and Comparison with other European Countries", $10^{\text {th }}$ International Conference on the European Energy Market - EEM13, Stockholm, May 2013.

[14] CENELEC, Technical Report: "CLC/TR 50555 Interruption Indexes", Brussels, May 2010.

[15] A. Falcão and M. Bollen, "Exceptional events and force majeure events and their use in the electricity sector," in Proc. CIRED International Conference on Electricity Distribution, Prague, June 2009. 